SOME RECENT RESULTS FROM UV OBSERVATIONS

\author{
M.J. Seaton, \\ Department of Physics and Astronomy, \\ University College London, Gower Street, London WClE 6BT
}

Abstract.

UV observations with IUE have led to recognition of the importance of di-electronic recombination in PN, for the calculation of ionization equilibria and for production of the features C II $\lambda 1335$ and C III $\lambda 2297$.

The determination of $C$ abundances is discussed. In high-excitation nebulae one must allow for absorption of C IV $\lambda 1549$ by dust. It has been shown that $C / 0 \leqslant 0.5$ for five $P N$ with a silicate IR feature and that $\mathrm{C} / 0 \approx 1$ for four with a SiC feature.

Dust opacities $\tau_{D}$ have been deduced from observed ratios (C IV $\lambda 1549) /($ C III $\lambda 2297$ ) and (C II $\lambda 1335) /(C$ I $\lambda 4267)$. The values obtained, $\tau_{D} \simeq 0.1$, are consistent with the observed strength of thermal infra-red emission.

Earlier UV observations of PN were made by Pottasch et al. (1978) using ANS. Results obtained using IUE support neither their downward revisions of star temperatures nor their claim that a number of stars with $T_{Z}(H e I I) ~ \gg T_{Z}(H I)$ are optically thick for $H I$.

From IUE observations it is concluded that the planetary $K 648$ in $M 15$ has $C / O=2.4$ and that its central star has a temperature of $30000 \mathrm{~K}$ and a luminosity of $1700 \mathrm{~L}_{\odot}$.

The present contribution is concerned with some IUE work with which I have been associated but makes no attempt at a comprehensive review of IUE observations of planetary nebulae. 
1. CARBON ABUNDANCES.

The determination of $C$ abundances is of interest because $C$ can be enriched by He burning. It is convenient to consider $\mathrm{C} / 0$ ratios, for which the solar value is $C / 0=0.6$ (Lambert, 1978). The determination of $\mathrm{C} / 0$ ratios is also of interest in connection with the interpretation of IR spectra, since objects with $\mathrm{C} / 0<1$ are expected to have IR features different from those in objects with $C / 0>1$ (see review by Barlow in the present volume). While we require $\mathrm{C} / 0$ ratios to an accuracy of much better than a factor of two, different methods in use a few years ago were giving results discrepant by as much as two orders of magnitude!

\subsection{C lines observed with IUE.}

IUE observations give fluxes in the collisionally-excited lines

C II] $\lambda 2326$, C III] $\lambda 1908$ and C IV $\lambda 1549$.

The $\mathrm{C}^{+} / \mathrm{C}^{2+}$ ratio for IC 418 obtained from $\lambda 2326$ and $\lambda 1908$ was found to be larger than had been predicted from ionization models, by a factor of 5 , and Harrington et al. (1980) suggested that $\mathrm{C}^{2+} \rightarrow \mathrm{C}^{+}$di-electronic recombination might be important. This was confirmed by Storey (1981 and review in the present volume) who shows that di-electronic recombination increases $\mathrm{C}^{+} / \mathrm{C}^{2+}$ and $\mathrm{C}^{2+} / \mathrm{C}^{3+}$ ratios and also leads to production of a number of spectrum lines, including

C II $\lambda 1335$ and C III $\lambda 2297$.

The C II $\lambda 1335$ multiplet is observed in IC 418 both using high dispersion. (Clavel et. al. 1981) and using low dispersion at a position offset from the central star (Adams and Seaton, 1982a). It is also observed in other objects but is generally weak due to absorption by interstellar C II. IC 418 is a favourable case, because it has a radial velocity which is sufficiently large to displace the wavelength of the C II emission from that of the interstellar C II absorption.

The C III $\lambda 2297$ line is observed in the spectra of a number of planetaries but is fairly weak and, in low-dispersion spectra, is blended with He II $\lambda 2307$ and partially blended with [0 III] $\lambda 2321$ and C II] $\lambda 2326$. For NGC 7009 and NGC 7662 results obtained from low-dispersion observations (Harrington et al. 1981) are confirmed by highdispersion observations (Harrington et al., 1981, and Flower et al. 1982 ).

\subsection{Resonance lines and dust absorption.}

C II $\lambda 1335$ and C IV $\lambda 1549$ are resonance multiplets and optical depths for scattering are of order $10^{4}$ for $\lambda 1335$ in low-excitation nebulae and for $\lambda 1549$ in high-excitation nebulae. Scattering of photons in resonance lines enhances the probability of their being absorbed by 
internal dust. The mean probability of photon escape will be denoted by $\mathrm{f}_{\mathrm{e}}$.

For IC 418 , Clavel et al. compared the ratio of fluxes

$$
F(\text { C II } \lambda 1335) / F(C \text { II } \lambda 4267) \text {, }
$$

corrected for interstellar absorption, with the ratio of emissivities

$$
\text { j(C II } \lambda 1335) / j(C \text { II } \lambda 4267) \text {, }
$$

calculated using effective recombination coefficients of Storey (1981) for $\lambda 1335$ and of Pengelly (1963) for $\lambda 4267$. They found (1.3) to be about half of (1.4) and concluded that $f_{e}=0.5$ for $\lambda 1335$ in IC 418 . paring

The best evidence for absorption of $\lambda 1549$ by dust comes from com-

$$
F(C \text { IV } \lambda 1549) / F(C \text { III } \lambda 2297)
$$

with

$$
\int j(C \text { IV } \lambda 1549) d V / \int j(C \text { III } \lambda 2297) d V,
$$

where the integrals in (1.6) are over the observed volume. Now the emissivities for $\lambda 1549$ and $\lambda 2297$ are both proportional to $\mathrm{Ne}_{\mathrm{N}}\left(\mathrm{C}^{3^{+}}\right)$and it follows that (1.6) depends only on the mean electron temperature, $\mathrm{T}_{\mathrm{e}}$ (C IV), of the region in which most of the carbon is $\mathrm{C}^{3^{+}}$. For NGC 7009 and 7662, Harrington et al. (1981, 1982) find (1.5) to be smaller than (1.6), calculated for any reasonable value of $T_{e}(C$ IV); they estimate that $f_{e} \simeq 0.3$ for $\lambda 1549$ in both of these objects. paring

Evidence for absorption of $\lambda 1549$ has also been deduced from com-

$$
F(C \text { IV } \lambda 1549) / F(C \text { III] } \lambda 1908)
$$

with

$$
\int j(C \text { IV } \lambda 1549) d V / \int j(C \text { III } \lambda 1908) d V,
$$

calculated using models (see, for example, the abstracts by Peña and Torres-Peimbert, and by Köppen and Wehrse, in the present volume). While persuasive, the evidence from (1.7) is less compelling than that from (1.5). Since the emissivity for $\lambda 1549$ is proportional to $\mathrm{Ne}_{\mathrm{N}}\left(\mathrm{C}^{3^{+}}\right.$) while that for $\lambda 1908$ is proportional to $\mathrm{Ne}_{\mathrm{N}}\left(\mathrm{C}^{2^{+}}\right)$, the ratio (1.8) is more sensitive to the structure of a model than is the ratio $(1.6)$. This point is illustrated by the work of Harrington et al. (1982) who give two models for MGC 7662 with different assumptions concerning the spatial distribution of density and the spectral distribution of stellar flux. 


\subsection{Results for C abundances.}

We can now understand why large discrepancies arose in earlier $C$ abundance determinations, and we can obtain results of greatly improved accuracy.

Abundances of $\mathrm{C}^{2+}$ were obtained by Torres-Peimbert and Peimbert (1977, TPP) from observations of C II $\lambda 4267$ interpreted using the effective recombination coefficient of Pengelly (1963), which Storey (1981) shows to be reliable. Although discussion continues as to whether mechanisms other than recombination contribute to excitation of $\lambda 4267$ (see review by Harrington in the present volume), the main source of error in the $\mathrm{C}^{2+}$ abundances of TPP was probably the use of earlier photographic measurements of the $\lambda 4267 \mathrm{flux}$, which tended to be too large and hence to lead to an over-estimate of the $\mathrm{C}^{2+}$ abundance.

Abundances of $\mathrm{C}^{3^{+}}$obtained by Pottasch, Wesselius and van Duinen (1978, PWD) from observations of C IV $\lambda 1549$ with ANS were under-estimated due to neglect of absorption of $\lambda 1549$ by dust. Both TPP and PWD used ionization correction factors from models which did not take account of di-electronic recombination in calculating total recombination rates. For nebulae of higher escitation, in which most of the carbon is $\mathrm{C}^{2+}$ or $\mathrm{C}^{3^{+}}$, TPP over-estimated the ratios $\mathrm{C} / \mathrm{C}^{2+}$ while PWD underestimated the ratios $\mathrm{C} / \mathrm{C}^{3^{+}}$. In consequence of these various sources of error, the $C$ abundances of TPP were in many cases much larger than those of PWD (this was discussed by Harrington et al., 1981).

For high-excitation nebulae, in which much of the $c$ is $\mathrm{c}^{3^{+}}$, it is difficult to obtain accurate $C$ abundances, unless one has observations of $\lambda 2297$ or sufficient confidence in predictions of models. For nebulae of medium excitation accurate results can be obtained from $\lambda 1908$ and $\lambda 2326$, given good estimates of $T_{e}$ (C II) and $T_{e}$ (C III), or from $\lambda 4267$ if the observations and interpretation can be trusted. The most accurate $C$ abundances are obtained from IUE observations of C II] $\lambda 2326$ and $10 \mathrm{II}] \lambda 2470$ in low-excitation nebulae, since the $\mathrm{C}^{+} / 0^{+}$ratio from these two lines is insensitive to $\mathrm{T}_{\mathrm{e}}$ and to reddening.

In a UCL-Durham collaboration, IUE observations have been made of nine planetary nebulae selected from those for which IR observations have been made (see Aitken and Roche, 1982); five show a silicate feature and four show SiC. They are all fairly low-excitation objects, with $\lambda 1549$ weak or absent. The $c / 0$ ratios are given in Table 1 and it is seen that all the objects with a silicate feature have $c / 0 \leqslant 0.5$ while those with a SiC feature have $\mathrm{C} / 0 \geqslant 1$. Further discussion is given by Barlow in this volume. 
Table 1.

\begin{tabular}{l} 
Objects with \\
\multicolumn{3}{l}{ Silicate feature } \\
$\begin{array}{lll}\text { Nebula } & \text { C/0 } & \text { Ref. } \\
\text { SwSt } 1 & 0.5 & \text { (a) } \\
\text { Hb } 12 & 0.3 & \text { (b) } \\
\text { IC } 4997 & 0.4 & \text { (c) } \\
\text { He 2-131 } & 0.3 & \text { (d) } \\
\text { M 1-26 } & 0.5 & \text { (e) }\end{array}$
\end{tabular}

$\begin{array}{lll}\frac{\text { Objects with }}{\text { SiC feature }} & \\ \frac{\text { Nebula }}{\text { Neb/0 }} & \text { Ref. } \\ \text { NGC 6572 } & 1.1 & \text { (f) } \\ \text { IC 2501 } & 0.9 & \text { (g) } \\ \text { NGC 6790 } & 0.8: & \text { (g) } \\ \text { IC 418 } & 1.3 & \text { (d) }\end{array}$

References: (a) Cohen, Flower and Goharji, this volume;

(b) Flower and Penn, this volume; (c) Flower (1980);

(d) Adams and Seaton (1982b); (e) Adams and Barlow, this volume; (f) Flower and Penn (1981); (g) Adams (1982)

\section{DUST OPACITIES.}

\subsection{Theory.}

The problem of radiative transfer with scattering in a spectrum line and with absorption has been solved by Hummer and Kunasz (1980) for plane parallel models. Figure l shows, for a mid-plane source, the escape probability $f_{e}$ against scattering optical depth $\tau_{s}$ for two values of dust optical depth, $\tau_{0}=0.1$ and 0.2 (these results are for a ratio of natural to Doppler widths of $a=4.7 \times 10^{-4}$, typical of values for planetary nebulae). It is of interest that, for $\log \left(\tau_{S}\right)$ between about 4.0 and $5.5, f_{e}$ increases as $\tau_{S}$ increases; this is because an increase in the scattering opacity can lead to a shorter path-length through the absorbing dust before escape occurs in the near line-wing.

\subsection{Determination of dust opacities.}

Harrington et al. (1982) construct a model of NGC 7662 which gives agreement with observations for $F(\lambda 2297) / F(\lambda 1908)$ and comparing the observed value of (1.5) with the calculated value of (1.6) they deduce that $f_{e}=0.3$ for $\lambda 1549$. It follows that the dust optical depth is $\tau_{D}=0.10$. From $f_{e}=0.5$ for $\lambda 1335$ in IC 418 , Clavel et al. obtain $\tau_{D}^{D}=0.08$. 


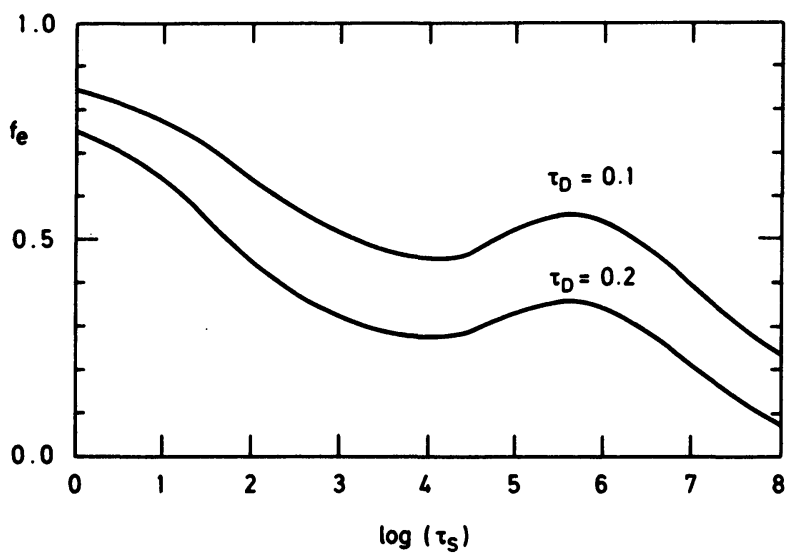

Figure 1. The escape probability $f_{e}$, for a mid-plane source, against scattering optical depth $\tau_{S}$ for two values of dust optical depth $\tau_{D}$ (from Hummer and Kunasz, 1980).

\subsection{Thermal infra-red emission.}

Let $F(L y \alpha)$ be the Ly $\alpha$ flux which would be observed if there were no absorption; it can be calculated using fluxes observed in other hydrogen lines. Let $F(T I R)$ be the total thermal infra-red emission, obtained from observations. Table 2 gives contributions to $F(T I R) / F(L y \alpha)$ calculated by Harrington et al. (1982) for NGC 7662, assuming that $\tau_{D}=0.1$ and that $\tau_{p}$ is independent of wavelength. The contributions are: absorption of C IV $\lambda 1549$; absorption of $L y \alpha$; absorption of radiation from the stellar continuum (giving a contribution to $F(T I R)$ of $F(\operatorname{star})\left\{1-\exp \left(-\tau_{D}\right)\right\}$ where $F(s t a r)$ is the total stellar flux). Adding these contributions gives a value of $F(T I R) / F(L y \alpha)$ in close agreement with that from the measurements of Moseley (1980), which provides a good check on the value used for $\tau_{D}$.

Table 2. Thermal infra-red emission in NGC 7662.

Contribution to $F(T I R) / F(L y \alpha)$

$\begin{array}{ll}\text { C IV } & 0.4 \\ \text { Lya } & 0.6 \\ \text { Star continuum } & 2.1 \\ \text { Total calculated } & \frac{3.1}{} \\ \text { Observed } & 2.7\end{array}$


2.4 The C IV doublet.

The collision strengths for excitation of the components of the C IV doublet, $\lambda 1548.2$ and $\lambda 1550.8$, are proportional to the statistical weights and, allowing for small differences in excitation energies, the ratio of emissivities is

$$
\dot{j}(\lambda 1548.2) / j(\lambda 1550.8)=2.00 \exp \left(-160 / T_{e}\right)
$$

with $\mathrm{T}_{\mathrm{e}}$ in $\mathrm{K}$. The oscillator strengths for the two lines are also in the 2 to 1 ratio of statistical weights and it is seen from Figure 1 that a change in $\tau_{S}$ by a factor of 2 gives only a small change in $f_{e}$. It follows that, even if there is absorption by dust, we would not expect the ratio of fluxes,

$$
R=F(\lambda 1548.2) / F(\lambda 1550.8)
$$

to differ significantly from the ratio of emissivities.

There has been some discussion about values of $R$ obtained from IUE observations (see abstracts by Peña and Torres-Peimbert, and by Feibelman, in the present volume). Care is needed in view of the instrument's rather limited dynamic range. Flower et al. (1982) obtained a high-dispersion spectrum of NGC 7662 with an exposure time optimised for the measurement of $R$ (long enough to give good signal-to-noise yet not so long as to give saturation or ITF truncation). Using extendedsource data-extraction procedures they obtained $R=1.92 \pm 0.03$ (error estimate only for noise) compared with 1.98 for the ratio of emissivities.

\section{STELLAR PHOTOMETRY.}

High accuracy is required if information about temperatures of central stars is to be deduced from optical and UV photometry. The first problem is that the flux distributions, dependence of $F_{\lambda}$ (star) on $\lambda$, are insensitive to temperature (for the hotter stars the Rayleigh-Jeans limit is approached). Subsidiary problems arise from the need to correct for interstellar absorption and to subtract nebular continuum fluxes from observed fluxes (which in some cases leads to severe cancellation). One can define a colour temperature, $T_{C}$, if the dependence of $F_{\lambda}$ (star) on $\lambda$ is the same as that of $B_{\lambda}\left(T_{C}\right)$. Alternatively, one can fit $F_{\lambda}$ (star) to fluxes of stellar atmosphere models.

Zanstra temperatures $T_{z}$ (H I) and $T_{Z}$ ( He II) have been used extensively for the placement of stars on the $H R$ diagram. It has usually been assumed that those stars with $T_{Z}$ (He II) $>T_{Z}(H$ I) have nebulae optically thin for $H$ I and that $T_{Z}$ (He II) provides the best estimate of their effective temperatures. It is important to establish whether these assumptions are consistent with optical and UV photometry. 
Using broad-band UV measurements from ANS, Pottasch et al. (1978, PWWFD) obtained values of $T_{C}$ for a number of stars which were smaller than $T_{Z}\left(H e\right.$ II) and closer to $T_{Z}(H I)$. They considered that there was a need for a downward revision of many star temperatures, which had "rather far reaching" consequences for the placement of the stars on the diagram.

Subsequent studies have been made using IUE, and more recent results from optical photometry, and several such studies are reported in the present volume. A program of work at UCL has involved the use of IUE spectra from the World Data Center supplemented with new IUE observations to correct for regions of saturation or of under-exposure. All raw data have been scrutinized and fluxes have been re-extracted with correction of ITF and other errors in previous extractions. Results are presented, at this Symposium, by Clegg and Seaton for 20 hotter stars and by Adams and Barlow for 9 cooler stars.

One result which emerges clearly is that, for a number of stars, PWWFD adopted temperatures which are much too low. The case of NGC 7662 is discussed in detail by Harrington et al.(1982) who show that the central star has a temperature much larger than the $48000 \mathrm{~K}$ adopted by PWW:D. Two other examples are: NGC 3242, for which $T_{Z}(H \mathrm{I})=46000 \mathrm{~K}$, $T_{Z}($ He II $)=93000 \mathrm{~K}$, PWWFD adopted $50000 \mathrm{~K}$ and Clegg and Seaton obtain $T_{C}=90000 \mathrm{~K}$; and NGC 1535 for which $T_{Z}(H ~ I)=28000 \mathrm{~K}, T_{Z}(\mathrm{He}$ II $)=$ $73000 \mathrm{~K}$, PWWFD adopted $40000 \mathrm{~K}$ and Clegg and Seaton obtain $\mathrm{T}_{\mathrm{C}}=$ 65000 K. A number of nebulae which PWWFD assumed to be opticafly thick for $H$ I are almost certainly optically thin.

\section{THE PLANETARY K 648 IN THE GLOBULAR CLUSTER M 15.}

The planetary nebula $K 648$ in the globular cluster M 15 is discussed by 0'Dell et al. (1964), Peimbert (1973) and Hawley and Miller (1978). From the angular diameter $\theta=3^{\prime \prime .5}$ measured by Johnson et al.(1979) one obtains an ionized mass of $\mathrm{M}\left(\mathrm{H}^{+}\right)=0.10 \mathrm{M}_{\odot}$ (in the Discussion following my talk it was noted that $\theta$ had been over-estimated by Johnson et al. and that a better estimate for $M\left(H^{+}\right)$is $\left.0.03 M_{\odot}\right)$.

Aurière et al. (1982) have made IUE observations of $K 648$ and have measured $U, B$ and $V$ for the stars in the IUE aperture. Using fluxes in C II] $\lambda 2326$ and C III] $\lambda 1908$ they obtain $C / 0=2.4$. This indicates that $C$ enrichment has occurred but the unusually large value of $C / 0$ does not indicate an unusually large amount of enrichment, since the 0 abundance is low.

The H I Zanstra temperature of the central star is $29000 \mathrm{~K}$ and the colour temperature from optical and UV observations is $33000 \mathrm{~K}$. Taking the effective temperature to be $30000 \mathrm{~K}$ and the distance of $\mathrm{M} 15$ to be $10 \mathrm{kpc}$, the luminosity of the central star is $1700 \mathrm{~L}_{\odot}$. Figure 2 shows $K 648$ on the $\log (L), \log (T)$ diagram for $M 15$; results for the cluster stars have been obtained from the $V,(B-V)$ diagrams of Sandage (1970) and of Aurière and Cordini (1981), assuming the stars to radiate 
like black-bodies.

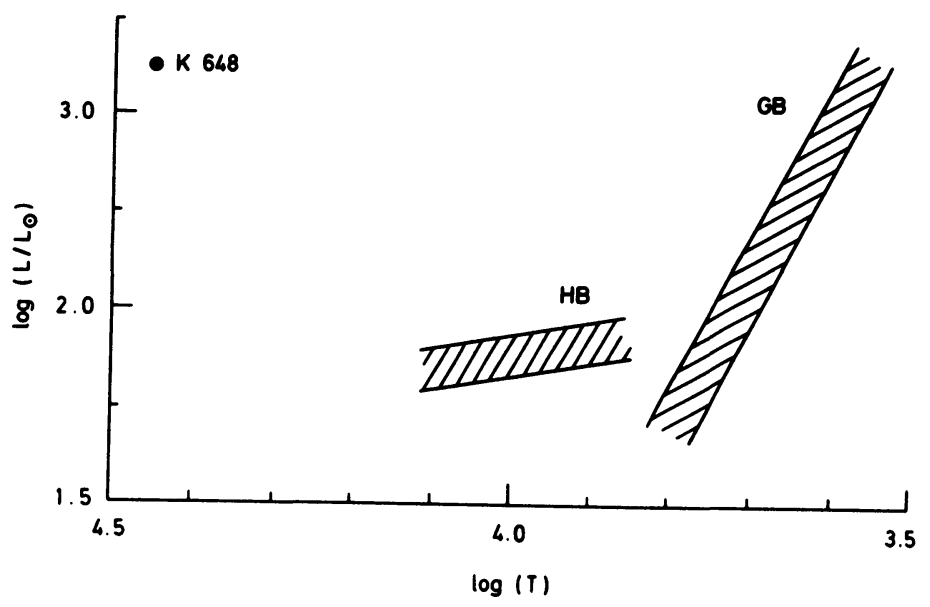

Figure 2. The $\log L$, $\log T$ diagram for $M 15$ showing $K 648$, the horizontal branch, $\mathrm{HB}$, and the giant branch, GB.

\section{References.}

1. Adams S, 1982, Mon. Not. R. astr. Soc., to be submitted.

2. Adams S and Seaton MJ, 1982a, Mon. Not. R. astr. Soc., to be submitted.

3. Adams S and Seaton MJ, 1982b, Mon. Not. R. astr. Soc., 200,7P.

4. Aitken DK and Roche PF, 1982, Mon. Not. R. astr. Soc., $\overline{200}, 217$

5. Aurière M, Adams S and Seaton MJ, 1982, Mon. Not. R. astr. Soc., to be submitted.

6. Aurière M and Cordoni J-P, 1981, Astr. Astrophys., 100, 307.

7. Clavel J, Flower DR and Seaton MJ, 1981, Mon. Not. R. astr. Soc., 197,301 .

8. Flower UR, 1980, Mon. Not. R. astr. Soc., 193, 511.

9. Flower DR and Pen CJ, 1981, Mon. Not. R. astr. Soc., 194, 13 P.

10. Flower DR, Penn CJ and Seaton MJ, 1982, Mon. Not. R. astr. Soc., in press.

11. Harrington JP, Lutz JH, Seaton MJ and Stickland DJ, 1980, Mon. Not. R. astr. Soc., 191, 13 .

12. Harrington JP, Lutz JH and Seaton MJ, 1981, Mon. Not. R. astr. Soc., 195, 21P.

13. Harrington JP, Seaton MJ, Adams S and Lutz JH, 1982, Mon. Not. R. astr. Soc., 199, 517.

14. Hawley SA and Miller JS, 1978, Astrophys. J. 220, 609.

15. Hummer DG and Kunasz PB, 1980, Astrophys. J., 236, 609

16. Johnson HM, Balick B and Thompson AR, 1979, Astrophys. J., 233, 919. 
17. Lambert DL, 1978, Mon. Not. R. astr. Soc., 182, 249.

18. Moseley H, 1980, Astrophys. J., 238, 892.

19. O'Dell CR, Peimbert M and Kinman TD, 1964, Astrophys. J., 140, 119.

20. Peimbert M, 1973, Mém. Soc. Roy. Sci. Liège, 5, 307.

21. Pengelly RM, 1963, Thesis, London (see also Seaton MJ, 1978, Planetary Nebulae (ed Y. Terzian), p.13l, Reidel).

22. Pottasch SR, Wesselius PR and van Duinen RJ, 1978, Astr. Astrophys. $70,629$.

23. Pottasch SR, Wesselius PR, Wu C-C, Fieten $H$ and van Duinen RJ, 1978, Astron. Astrophys., 62, 95.

24. Sandage A, 1970, Astrophys. J., 162, 841.

25. Storey PJ, 1981, Mon. Not. R. astr. Soc., 195, 27 P.

26. Torres-Peimbert $S$ and Peimbert M, 1977, Rev. Mex. Astron. y. Astrophys. 2, 181 .

NUSSBAUMER: I have two comments: (1) observers find that in symbiotic stars the C IV doublet ratio is often not 2 : 1 , but smaller (e.g. Nussbaumer and Schild, 1981, Astron. Astrophys. 101, 118).

Kind1 and Nussbaumer have followed up the work of Flower on IC 4997 and confirm his findings: $\mathrm{C} / 0 \approx 0.5$ and 1 ow $\mathrm{C} / \mathrm{H}$ and $0 / \mathrm{H}$ abundance ratios. In addition, we find $\mathrm{N}$ to be underabundant, relative to the Sun, by a factor 5 and $\mathrm{Si}$ to be underabundant by a factor of more than 100.

SEATON: As far as I know, the doublet ratio could be almost anything in symbiotic stars! I think it very unlikely to much differ from 2 for emission from PN envelopes. For these cases, it is necessary to make a careful check on observational errors where values significantly different from 2 are obtained.

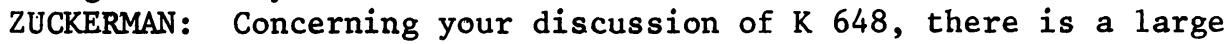
discrepancy between the momentum carried by the radiation field of the central star and that of the ionized gas (assuming a typical PN expansion velocity), in the sense that radiation pressure is inadequate to drive the expansion of the nebula. Therefore, either the central star was much more luminous in the recent past, or radiation pressure is not the mechanism that drives the outflow. The momentum discrepancy is sufficiently large to remain even if the ionized mass $\left(\approx 0.1 \mathrm{M}_{\odot}\right)$ that you mentioned is several times over estimated.

KAHN: In connection with Zuckerman's remark, the hot stellar wind will shock against the nebular gas and then be bottled up at a temperature $\approx 10^{6} \mathrm{~K}$. This confined gas will exert a much larger pressure on the nebula than the radiation directly from the star.

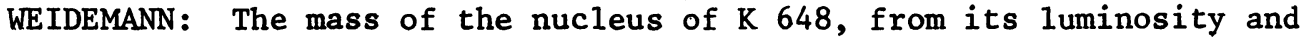
the core mass / luminosity relation, is $0.55 \mathrm{M}$. It cannot have been higher on the AGB, since $\mathrm{K} 648$ is population II, for which we expect exactly this final mass. Do you know the radius of the nebula, in order to check that the nucleus fits the Schönberner track? 
SEATON: The distance of M15 is about $10 \mathrm{kpc}$ and the angular diameter I quoted was 3.5 arc sec.

SCOTT: I have a comment on the radio flux density of $K 648$. Birkinshaw et al. have measured $4.0 \pm 1.7 \mathrm{~m} \mathrm{Jy}$ with the Cambridge $5 \mathrm{~km}$ telescope - a quarter of the early VLA measurement. The source was also unresolved ( $\leqslant 2$ arc sec).

POTTASCH: The VLA measurements of Johnson et al. were made with the early, incomplete array. More recent measurements show that their flux (16 m Jy) was overestimated and should be about $3.5 \mathrm{~m} \mathrm{Jy}$. Likewise, the diameter was overestimated. The main body of the nebula is probably not larger than 1 arc sec, and most of the emission is within a diameter of 0.7 arc sec. The nebular mass is $2 \times 10^{-2}$ to $3 \times 10^{-2} \mathrm{M}$.

IBEN: The $1{ }^{\circ}$ wer 1 imit to the luminosity of the carbon stars in the Magellanic Clouds is approximately $3 \times 10^{3} \mathrm{~L}_{0}$. One would expect this to map into a lower limit to the plateau luminosity of PN nuclei progeny. Is there any possibility that your estimate of $1.7 \times 10^{3} \mathrm{~L}_{\odot}$ could actually be larger by a factor 2 ?

SEATON: I think that revision by a factor 2 is unlikely but I could not exclude the possibility. 